\title{
Downregulation of miR-193b in systemic sclerosis regulates the proliferative vasculopathy by urokinase-type plasminogen activator expression
}

\author{
Naoki Iwamoto, ${ }^{1,2}$ Serena Vettori, ${ }^{1}$ Britta Maurer, ${ }^{1}$ Matthias Brock, ${ }^{1}$ Elena Pachera, ${ }^{1}$ \\ Astrid Jüngel, ${ }^{1}$ Maurizio Calcagni, ${ }^{3}$ Renate E Gay, ${ }^{1}$ Michael L Whitfield, ${ }^{4}$ \\ Jörg H W Distler, ${ }^{5}$ Steffen Gay, ${ }^{1}$ Oliver Distler ${ }^{1}$
}

\section{Handling editor Tore K Kvien}

- Additional material is published online only. To view please visit the journal online (http://dx.doi.org/10.1136/ annrheumdis-2014-205326)

${ }^{1}$ Division of Rheumatology, Center of Experimental Rheumatology, University Hospital and Zurich Center of Integrative Human Physiology (ZIHP), Zurich, Switzerland 2Unit of Translational Medicine, Department of Immunology and

Rheumatology, Nagasaki University Graduate School of Biomedical Sciences, Nagasaki, Japan

${ }^{3}$ Division of Plastic Surgery and Hand Surgery, University Hospital Zurich, Zurich, Switzerland

${ }^{4}$ Department of Genetics, Geisel School of Medicine at Dartmouth, Hanover, New Hampshire, USA

${ }^{5}$ Department of Internal Medicine 3, Institute for Clinical Immunology, University of Erlangen-Nuremberg, Erlangen, Germany

\section{Correspondence to} Dr Oliver Distler, Division of Rheumatology, University Hospital Zurich, Gloriastr. 25, Zürich 8091, Switzerland; Oliver.Distler@usz.ch

Received 31 January 2014 Revised 23 October 2014 Accepted 24 October 2014 Published Online First 10 November 2014

\section{(a) CrossMark}

To cite: Iwamoto $\mathrm{N}$, Vettori S, Maurer B, et al. Ann Rheum Dis 2016;75:303-310

\section{ABSTRACT}

Objectives To investigate the role of microRNA-193b$3 p$ (miR-193b) in the vascular pathophysiology of systemic sclerosis (SSc).

Methods Expression of miR-193b in skin biopsies and fibroblasts from patients with SSC and normal healthy $(\mathrm{NH})$ controls were determined by real-time PCR.

Transfection with miR-193b precursor and inhibitor were used to confirm targets of miR-193b. Proliferative effects of urokinase-type plasminogen activator (UPA) were determined by water-soluble tetrazolium salt- 1 assay and by analysis of proliferating cell nuclear antigen expression. Fluorescence activated cell sorting analysis was performed to investigate the effect of UPA on apoptosis. For inhibition of the UPA-cellular receptor for UPA (UPAR) pathway, UPAR neutralising antibodies and low molecular weight uPA were used.

Results We found that miR-193b was downregulated in SSc fibroblasts and skin sections as compared with $\mathrm{NH}$ controls. The expression of miR-193b was not affected by major profibrotic cytokines and hypoxia. Induction of miR-193b in SSc fibroblasts suppressed, and accordingly, knockdown of miR-193b increased the levels of messenger RNA and protein for UPA. UPA was found to be upregulated in SSC as compared with $\mathrm{NH}$ controls in a transforming growth factor- $\beta$ dependent manner, and UPA was strongly expressed in vascular smooth muscle cells in SSc skin section. Interestingly, UPA induced cell proliferation and inhibited apoptosis of human pulmonary artery smooth muscle cells, and these effects were independent of UPAR signalling.

Conclusions In SSc, the downregulation of miR-193b induces the expression of UPA, which increases the number of vascular smooth muscle cells in an UPARindependent manner and thereby contributes to the proliferative vasculopathy with intimal hyperplasia characteristic for SSC.

\section{INTRODUCTION}

Systemic sclerosis (SSc) is a multisystem connective tissue disease characterised by fibrosis of the skin and internal organs and by a widespread vasculopathy associated with high morbidity and mortality. ${ }^{1}$ In early stages of the disease, microvascular changes such as capillary dilatation with subsequent rarefaction occur. ${ }^{2} 3$ In addition to capillary rarefaction, another feature of the SSc vasculopathy is intimal hyperplasia of small arteries, which results from increased proliferation and decreased apoptosis of vascular smooth muscle cells (VSMCs). ${ }^{4} \quad 5$ Together, these vascular changes reduce blood flow and contribute to life-threatening clinical manifestations such as pulmonary arterial hypertension.

Although the detailed pathogenesis of the SSc vasculopathy still remains unclear, several key pathways have been identified. One such pathway is the urokinase-type plasminogen activator (uPA) and the cellular receptor for uPA (uPAR) pathway. uPA is a serine protease that initiates the conversion of plasminogen to plasmin, and it is also involved in the activation of metalloproteinase and other proteins by enzymatic cleavage of precursors. uPA can trigger cellular responses such as cell migration, differentiation and proliferation on several cell types via uPA-uPAR dependent and uPAR-independent pathways. ${ }^{6-9}$ Moreover, there is evidence that uPA contributes to neointimal growth and vascular remodelling. ${ }^{9-11}$

Recent key studies have shown that the uPA-uPAR pathway is impaired in SSc. Matrix metalloproteinase-12, which is overproduced by SSc microvascular endothelial cells (MVEC) and SSc fibroblasts, cleaves uPAR on the surface of MVEC, and this modification contributes to the impaired angiogenesis of SSc. ${ }^{12}{ }^{13}$ Accordingly, uPAR-/- mice develop increased apoptosis in endothelial cells in the dermis. ${ }^{14}$

MicroRNAs (miRNAs) are small non-coding RNAs, which regulate gene expression posttranscriptionally. ${ }^{15}{ }^{16}$ miRNAs repress protein synthesis by destabilising target mRNAs or inhibiting mRNA translation through base-pairing with $3^{\prime}$ untranslated region of target mRNAs. ${ }^{17}$ There is growing evidence that the deregulation of miRNA is implicated in the pathogenesis of human diseases, ${ }^{18-20}$ and miRNAs are thought to represent novel disease biomarkers and potential therapeutic targets. ${ }^{21}$ Altered expression of miRNAs has also been reported in human autoimmune diseases including SSc. ${ }^{22-24}$ However, the role of miRNAs in the pathogenesis of vasculopathy in SSc has not been addressed.

In the present study, we found that microRNA-193b-3p (miR-193b) was downregulated in cultured fibroblasts and skin biopsies of patients with SSc. Furthermore, we showed that the downregulation of miR-193b results in an overexpression of its target uPA. Increased levels of uPA 
contributed to the proliferative vasculopathy of SSc by inhibiting apoptosis and inducing proliferation of VSMCs in an uPAR-independent manner.

\section{MATERIALS AND METHODS}

More detailed information on methods is provided in the online data supplement.

\section{Patients, biopsy specimens and cell culture}

Skin biopsy and skin fibroblasts were obtained from patients with SSc ( $n=33,5$ diffuse SSc, 28 limited SSc) and normal healthy $(\mathrm{NH})$ donors $(\mathrm{n}=25)$. All patients fulfilled the criteria for SSc as described by LeRoy et al. ${ }^{25}$ The study was approved by the local ethics committees. Primary human pulmonary artery smooth muscle cells (HPASMCs) were purchased from Invitrogen (Basel, Switzerland).

\section{Reagents and stimulation assays}

Dermal fibroblasts and/or HPASMCs were stimulated with transforming growth factor- $\beta$ (TGF- $\beta$ ), platelet-derived growth factor B (PDGF-B), interleukin $1 \beta$ (IL-1 $\beta$ ) (all R\&D Systems, Abingdon, UK), high molecular weight uPA (HMW-uPA) (Loxo, Dossenheim, Germany), low molecular weight uPA (LMW-uPA) (Loxo), tumour necrosis factor (TNF)-related apoptosis-inducing ligand (TRAIL) (R\&D Systems) or neutralising monoclonal antibodies against human uPAR (American Diagnostica, Pfungstadt, Germany). Carrier solution of stimulants was added at a similar volume for control experiments. Exposure to hypoxia was done as described. ${ }^{26}$

\section{Transfection experiments}

Dermal fibroblasts or HPASMCs were transfected with synthetic precursor miRNA (pre-miR), with inhibitors of miR-193b (anti-miR), or with scrambled controls (pre-miR/anti-miR negative control \#1; Ambion/Applied Biosystems, Rotkreuz, Switzerland) with the use of Lipofectamine 2000 reagent (Invitrogen). Transfection efficiency was controlled by TaqMan real-time PCR.

\section{Fluorescence activated cell sorting (FACS) analysis}

For analysis of apoptosis, cells were detached with accutase and stained for annexin $\mathrm{V}$ and propidium iodide (PI) with the annexin V-FLUOS staining kit (Roche, Mannheim, Germany). Cells were subsequently analysed on the FACSCalibur flow cytometer. Apoptotic cells were defined as PI-negative (indicating an intact plasma membrane) and annexin V-fluorescein isothiocyanate (FITC)-positive. ${ }^{27}$

\section{RNA isolation and quantitative real-time PCR analysis}

RNA was isolated from fibroblasts and fresh frozen skin tissue with the mirVana Isolation Kit (Ambion/Applied Biosystems) and from paraffin embedded sections with Recover All Total Nucleic Acid Isolation Kit (Ambion/Applied Biosystems). Reverse transcription and TaqMan real-time PCRs (for miRNAs) or SYBR green real-time PCR were performed as described. ${ }^{24}$

\section{Western blot analysis, cell proliferation assay by WST-1 and immunohistochemistry}

Western blots were performed using polyclonal antibodies against uPA (Abcam, Cambridge, UK), monoclonal antibodies against proliferating cell nuclear antigen (PCNA) (Imgenex, San Diego, California, USA) or monoclonal antibodies against $\alpha$-tubulin (Abcam) according to standard protocols. ${ }^{28}$ Cell proliferation was measured using water-soluble tetrazolium salt (WST-1)/electro coupling solution (Abcam) as recommended by the manufacturer. Immunohistochemistry was performed using polyclonal rabbit anti-human uPA antibodies (Abcam), and monoclonal mouse antibodies against $\alpha$-smooth muscle actin ( $\alpha$-SMA) (Sigma-Aldrich. Buchs, Switzerland). Irrelevant isotypematched IgGs were used as controls. Staining was visualised with 3-amino-9-ethylcarbazole, diaminobenzidine or by fast blue using an alkaline phosphatase substrate kit (all from Vector, Burlingame, USA).

\section{Statistical analysis}

Data are expressed as mean and SEM. Normal distribution of data was confirmed using the Kolmogorov-Smirnov test. Statistical significance was evaluated by Student's paired and unpaired $t$ test. For non-parametric data, the Mann-Whitney $\mathrm{U}$ test and Wilcoxon matched-pairs signed rank test were used. $\mathrm{p}<0.05$ was considered statistically significant.

\section{RESULTS}

\section{Downregulation of miR-193b in SSc}

In our preliminary experiments using the TaqMan Human MicroRNA Array (Ambion/Applied Biosystems), we found that miR-193b was one of the most significantly and most consistently downregulated miRs in dermal SSc fibroblasts compared with NH fibroblasts (not shown). To validate the microarray findings, quantitative real-time PCR with additional cultured fibroblasts was performed. In agreement with the array data, there was a significant difference in the expression of miR-193b between SSc fibroblasts and healthy control fibroblasts. As shown in figure 1A, the baseline levels of miR-193b were consistently downregulated in SSc fibroblasts to $31 \pm 9.6 \%(\mathrm{p}<0.001)$.

Next, we investigated the expression of miR-193b in skin samples from patients with SSc. First, the expression of the miR-193b in tissue extracts isolated from paraffin-embedded skin sections was analysed, and similar to the findings in SSc fibroblasts, decreased levels of the miR-193b were observed. MiR-193b was downregulated in the dermis of patients with $\mathrm{SSc}$ as compared with healthy controls to $54 \pm 7 \% \quad(\mathrm{p}<0.01$, figure 1B).

To exclude the possibility that paraffin processing influenced the content of miRNA in the tissue, we additionally investigated the expression of the miR-193b in fresh frozen skin biopsy samples. Consistent with the previous results, miR-193b was found to be downregulated to $62 \pm 20 \%(p=0.087)$ in patients with SSc as compared with healthy controls (figure 1C).

MiRs from the same cluster often show overlapping regulations and downstream effects. We therefore analysed the baseline levels of miR-365, which is the other member of the miR-193b-365 cluster. $^{29}$ MiR-365 was also downregulated in cultured SSc fibroblasts, while no differences were detected in fresh frozen and paraffin embedded skin biopsies (not shown).

\section{Downregulation of miR-193b is independent of profibrotic cytokines and hypoxia}

To explore the factors that potentially downregulate the levels of miR-193b in SSc, we analysed the effects of major profibrotic cytokines and hypoxia. We first stimulated dermal SSc and $\mathrm{NH}$ fibroblasts with key profibrotic cytokines including IL-1 $\beta$, TGF- $\beta$ and PDGF-B for various time points $(12 \mathrm{~h}, 24 \mathrm{~h}, 48 \mathrm{~h}$ and 5 days) and at different concentrations considered physiological in humans. None of these stimuli had any influence on the expression of miR-193b (see online supplementary figure S1). Similarly, exposure to hypoxia, which is thought to independently contribute to the progression of fibrosis, ${ }^{30}$ had no consistent effects on the expression of miR-193b compared with 
Figure 1 Expression of microRNA-193b (miR-193b) in patients with systemic sclerosis (SSc) and healthy controls, as determined by TaqMan-based real-time PCR. Expression of miR-193b in samples of patients with SSc was determined relative to healthy controls, which was defined as $100 \%$. (A) miR-193b was markedly reduced in cultured SSc skin fibroblasts $(n=12)$ as compared with healthy controls $(n=6)$. (B) Similar to the in vitro findings, the expression miR-193b in paraffin-embedded skin sections from patients with SSC $(n=5)$ was downregulated as compared with healthy controls $(n=5)$. (C) Expression of miR-193b in snap frozen skin biopsy samples from patients with SSC $(n=9)$ was downregulated as compared with healthy controls $(n=5)$. Values are the mean and SEM. ${ }^{*} p<0.05$ versus healthy controls.

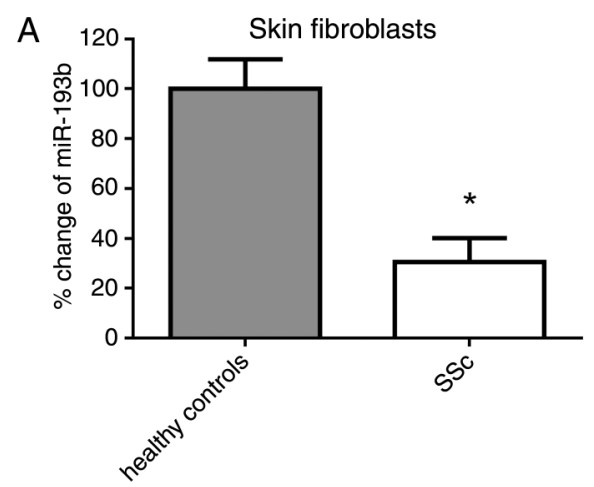

B 140 Paraffin embedded skin sections

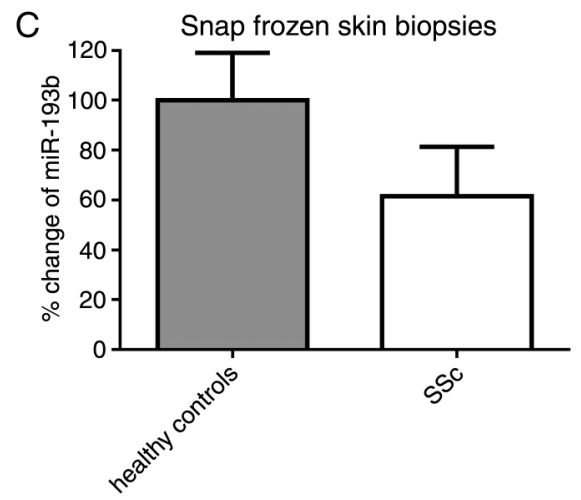

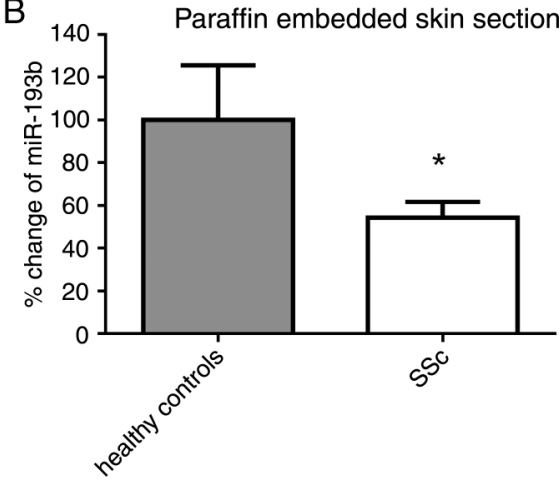

normoxic controls (see online supplementary figure S1). These results suggest that downregulation of miR-193b might be independent of these major profibrotic cytokines and hypoxia in SSc.

\section{uPA is targeted by miR-193b in SSc fibroblasts and HPASMC}

To elucidate the functional consequences of the downregulation of miR-193b in SSc, we searched for potential gene targets of miR-193b by performing in silico studies using the miRecords (http://mirecords.biolead.org/). The predicted potential targets including uPA, collagen type VI $\alpha 2$, TGF $\beta$ receptor III, insulinlike growth factor binding protein 5 , Calmodulin 1 , WinglessInt (WNT) 1 inducible signalling pathway protein 1 and plateletderived growth factor $\alpha$ (PDGFA) were further analysed by gain and loss of function assays with miR-193b.

In SSc dermal fibroblasts, transfection with pre-miR-193b increased the levels of miR-193b by $349 \pm 522$-fold as compared with scrambled controls. Knockdown with anti-miR-193b reduced the expression of miR-193b to $0.03 \pm 0.03$-fold. Transfections did not substantially alter the levels of miR-365 indicating successful and specific transfection.

Among the predicted targets, uPA showed the most consistent and significant results in the gain and loss of function assays and were further analysed. Overexpression of miR-193b reduced the expression of uPA to $0.41 \pm 0.12$-fold $(p<0.05)$ on the mRNA level (figure 2A). Consistently, knockdown of miR-193b increased the expression of uPA by $3.76 \pm 0.93$-fold $(p<0.05)$ on the mRNA level (figure 2B). Similar results were obtained with NH dermal fibroblasts (not shown) and HPASMCs (see online supplementary figure S2). These findings were confirmed on the protein level by western blotting (figure 2C, D). Densitometry revealed that overexpression of miR-193b reduced the expression of uPA protein by $0.56 \pm 0.05$-fold $(\mathrm{p}<0.05)$ and knockdown of miR-193b increased the expression of $\mathrm{uPA}$ by $2.93 \pm 0.95$-fold $(\mathrm{p}=0.0625)$. Taken together, these findings confirm uPA as a target of miR-193b in SSc fibroblasts.

\section{Overproduction of uPA in SSc}

Next, we investigated whether uPA is upregulated in SSc. First, we compared the basal expression of uPA in SSc fibroblasts with healthy control fibroblasts. Baseline levels of uPA mRNA were not significantly changed compared with healthy control fibroblasts $(3.58 \pm 1.58$-fold, $\mathrm{p}=0.43)$. On the protein level, basal levels of uPA were slightly higher than in healthy controls without reaching statistical significance $(n=5,1.76 \pm 0.41$-fold, $\mathrm{p}=0.14$ ) (figure $3 \mathrm{~B}$ ). To simulate the profibrotic conditions present in SSc in vivo, fibroblasts were stimulated with TGF- $\beta$. Indeed, in TGF- $\beta$ stimulated fibroblasts, levels of uPA mRNA were strongly increased in SSc fibroblasts as compared with healthy control fibroblasts $(8.5 \pm 3.91$-fold, $\mathrm{p}<0.05$, figure $3 \mathrm{~A})$.

We next analysed the expression of uPA using a recently published microarray data set derived from skin biopsies from distinct scleroderma subsets including 17 patients with diffuse SSc, 7 patients with limited SSc, 3 patients with morphoea and 6 healthy controls. ${ }^{31}$ Analysis of this microarray revealed increased expression of uPA mRNA in the inflammatory subset of SSc, while it was expressed at low levels in normal-like subset that included all healthy controls (average fold change $2.59 \pm 0.47 ; \mathrm{p}=0.000146$ by $\mathrm{t}$ test). Taken together, these data show that there is an overexpression of uPA in SSc, which is related to TGF- $\beta$.

\section{Localisation of uPA expression in human SSc skin sections}

We next investigated the in situ expression of uPA in SSc skin biopsies by immunohistochemistry. Weak expression of uPA protein was seen in fibroblast-like cells, but pronounced expression of uPA protein was found in vascular structures (figure 4A). To confirm that uPA was preferentially expressed in vessels, we performed double staining with uPA and the VSMC marker $\alpha$-SMA. Double staining showed that uPA-positive cells were double stained with $\alpha$-SMA, suggesting that uPA is preferentially expressed by VSMCs in small arteries in SSc (figure 4B). 
A

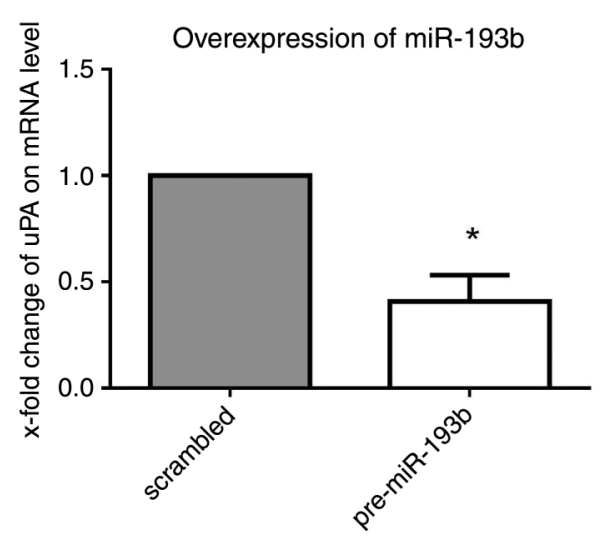

C

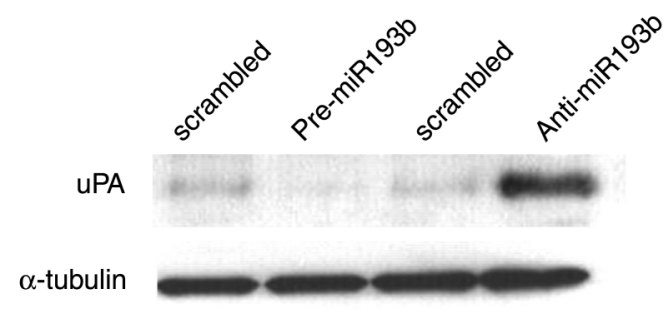

B

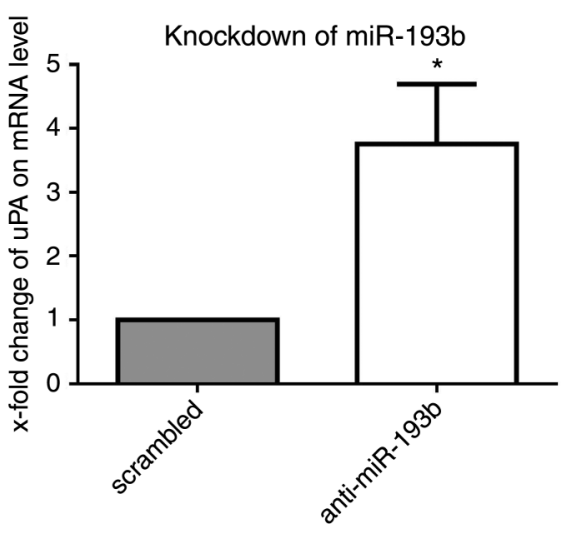

$\mathrm{D}$

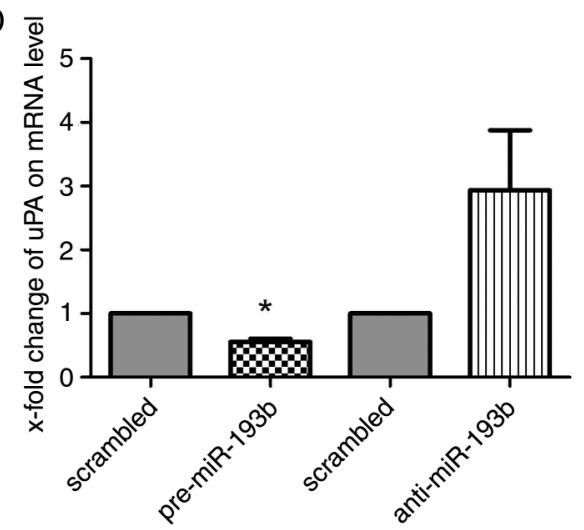

Figure 2 Influence of overexpression and knockdown of miR-193b on the expression of urokinase-type plasminogen activator (uPA). Expression of uPA in systemic sclerosis (SSc) fibroblasts was determined relative to the controls transfected with scrambled RNA, which was defined as 1. (A) At the mRNA level, transfection of SSc fibroblasts $(n=8)$ with precursor miR-193b (pre-miR-193b) for $72 \mathrm{~h}$ as compared with scrambled RNAtransfected controls $(n=8)$ decreased the levels of uPA, as determined by SYBR green real-time PCR analysis. (B) At the mRNA level, knockdown of miR-193b for $72 \mathrm{~h}$ in SSc fibroblasts $(n=8)$ as compared with scrambled RNA-transfected controls $(n=8)$ increased the levels of uPA, as determined by SYBR Green real-time PCR. (C) At the protein level, overexpression of miR-193b for $36 \mathrm{~h}$ in SSc skin fibroblasts $(n=5)$ decreased the expression of uPA as compared with scrambled RNA-transfected controls $(n=5)$, and knockdown of miR-193b for $36 \mathrm{~h}$ in SSc skin fibroblasts $(n=5)$ increased the expression of uPA as compared with scrambled RNA-transfected controls $(n=5)$, as determined by western blotting. Representative results are shown. (D) Semiquantitative analysis was performed by densitometry. Values are given as means \pm SEM. In the western blots, $\alpha$-tubulin was used as loading control. * $\mathrm{p}<0.05$ versus scrambled RNA-transfected controls.

\section{UPA induces proliferation and suppresses apoptosis on HPASMC}

The localisation of uPA in SSc skin sections suggested that uPA might play a critical role in the vascular pathology of SSc. To assess whether uPA contributes to the proliferative vasculopathy of SSc, we first examined the proliferative effects of uPA on HPASMCs by WST-1 assay and PCNA protein expression. Cells were stimulated with various concentrations of HMW-uPA for $24 \mathrm{~h}$ and cell viability was assessed by using the WST-1 method, an indicator of active cell metabolism. HMW-uPA increased the proliferation of HPASMC by $21.75 \pm 7.5 \%(10 \mathrm{nM}, \mathrm{p}<0.004$, figure $5 \mathrm{~A}$ ). This finding was confirmed by the observed expression of PCNA protein, another marker of proliferation. Consistent with the WST-1 assay, PCNA expression was increased by HMW-uPA $(1.5 \pm 0.16$-fold, $\mathrm{p}<0.03$, figure $5 \mathrm{~B})$.

Because the proliferative vasculopathy of SSc is characterised by enhanced proliferation, and suppressed apoptosis of VSMCs, ${ }^{32} 33$ we next assessed the effect of uPA on apoptosis. To detect apoptosis, annexin V-PI double staining was performed and analysed by flow cytometry. As shown in figure $5 \mathrm{C}$, HPASMC stimulated with $5 \mathrm{nM}, 10 \mathrm{nM}, 25 \mathrm{nM}$ of HMW-uPA showed a decrease of $17 \%, 33 \%, 21 \%$ in the number of apoptotic cells, respectively $(\mathrm{p}<0.0001,10 \mathrm{nM}$ stimulation experiment). We also examined the effect of uPA on TRAIL-induced apoptosis. Similar to spontaneous apoptosis, stimulation with 5 $\mathrm{nM}, 10 \mathrm{nM}, 25 \mathrm{nM}$ of HMW-uPA in combination with TRAIL decreased the number of apoptotic cells by $17 \%, 48 \%$ and $18 \%$, respectively $(\mathrm{p}<0.02,10 \mathrm{nM}$ stimulation experiment).

These results suggest that overproduction of uPA in SSc increases the number of VSMCs via suppression of apoptosis and increase of proliferation resulting in hyperplasia of VSMCs characteristic for the proliferative vasculopathy in SSc.

\section{uPA suppressed apoptosis of HPASMC via UPA receptor-independent pathways}

In SSc, receptor signalling via uPAR is impaired because of cleavage of uPAR by overproduction of matrix metalloproteinase-12..12 13 Thus, we next wanted to investigate whether the observed antiapoptotic effects of uPA on HPASMC are independent of uPAR. First, we performed blocking experiments for uPAR using uPAR neutralising antibodies. Indeed, uPA inhibited spontaneous and TRAIL-induced apoptosis even in the presence of uPAR neutralising antibodies (figure 6A). To confirm these results with an independent method, we stimulated HPASMC with LMW-uPA, which in contrast to HMW-uPA lacks the amino-terminal fragment and therefore does not bind to uPAR. ${ }^{34} \mathrm{LMW}-\mathrm{uPA}$ inhibited spontaneous as well as TRAIL-induced apoptosis on HPASMC to a similar extent as 
A

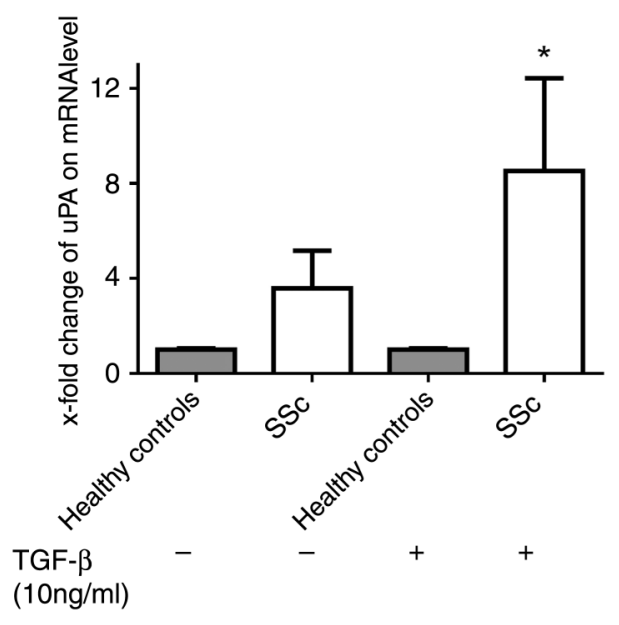

B
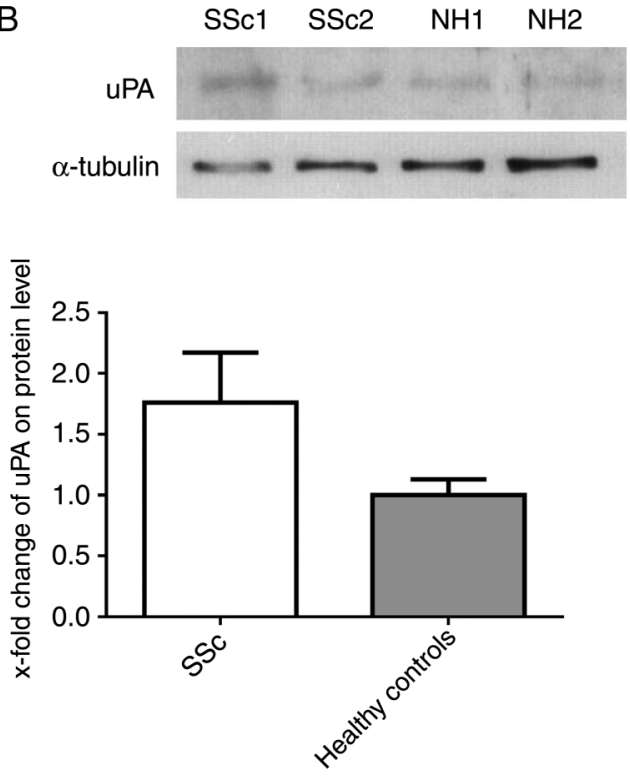

Figure 3 Expression of urokinase-type plasminogen activator (UPA) in dermal fibroblasts from systemic sclerosis (SSc) skin and healthy controls. Expression of uPA in SSc fibroblasts was determined relative to the healthy controls, which was defined as 1. (A) On the mRNA level, expression of uPA was slightly, but not significantly increased in SSc skin fibroblasts $(n=9)$ in comparison with healthy controls $(n=6)$, as determined by SYBR green real-time PCR. However, after stimulation with transforming growth factor- $\beta$ (TGF- $\beta$ ), the expression of uPA was markedly increased in SSC skin fibroblasts $(n=10)$ as compared with healthy controls $(n=6)$. (B) On the protein level, expression of uPA was slightly increased in SSc skin fibroblasts $(n=5)$ as compared with healthy controls $(n=4)$, as determined by western blotting (top), confirming the results from the mRNA analysis. Two representative experiments are shown. The semiquantitative analysis was performed by densitometry (bottom). Values are given as the means \pm SEM. In the western blots, $\alpha$-tubulin was used as loading control. * $p<0.05$ versus healthy controls.

with HMW-uPA (figure 6B). These results suggest that uPA inhibits apoptosis in HPASMC via uPA-uPAR independent pathways.

\section{DISCUSSION}

Our study suggests a crucial role of miR-193b in the pathophysiology of SSc (see online supplementary figure S3). We have

A
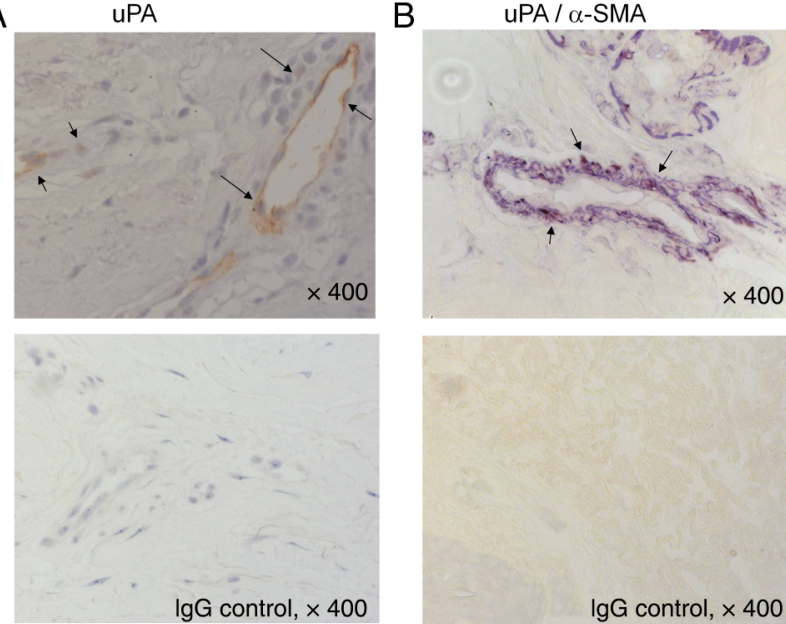

Figure 4 Urokinase-type plasminogen activator (uPA) expression in the skin of patients with systemic sclerosis (SSC). (A) Representative sections of SSc skin stained for UPA or IgG control. Positive staining of uPA appears as light brown colour. Expression is seen in vascular structures (arrows). Sections were counterstained with eosin. (B) SSC skin section double-stained for uPA (purple) and $\alpha$-smooth muscle actin ( $\alpha$-SMA) (blue) or IgG control. Co-staining of uPA and $\alpha-S M A$ shows expression of UPA in vascular smooth muscle cells (arrows). One representative section is shown $(n=5)$. Original magnification $\times 400$. shown that the expression of miR-193b is decreased in SSc compared with $\mathrm{NH}$ controls, and that downregulation of miR-193b leads to increased production of uPA. Because (1) uPA was strongly expressed by VSMCs, (2) VSMCs are cellular key players in the pathophysiology of SSc-pulmonary arterial hypertension and (3) uPA has been described as a proliferative factor in several cell types, ${ }^{6-9}$ we next focused on the role of uPA on HPASMC. We could show that uPA is involved in the pathophysiology of proliferative vasculopathy via induction of cell proliferation and inhibition of apoptosis. These results suggest that uPA is an important player in the pathophysiology of the proliferative vasculopathy in SSc.

There is growing evidence that uPA is involved in the pathophysiology of SSc. Landmark studies by D'Alessio et al ${ }^{12}$ showed that the constitutive overproduction and secretion of matrix metalloproteinase-12 by SSc dermal MVECs and fibroblasts cause endothelial uPAR cleavage resulting in impaired angiogenesis. ${ }^{13}$ These findings suggest that the uPA-uPAR pathway is impaired in SSc. Thus, the increased levels of uPA mediated by decreased levels of miR-193b found in our study must exert their effects on the intimal hyperplasia mainly via uPAR-independent pathways. Indeed, our uPAR blocking experiments using uPAR neutralising antibodies, siRNA against uPAR as well as LMW-uPA demonstrated that uPAR-independent pathways play a major role in the antiapoptotic effects of uPA on HPASMCs. This creates a novel, complex dual role for uPA in the pathophysiology of SSc with (1) impaired angiogenesis and increased apoptosis due to the lack of functional UPAR on MVEC and (2) proliferation and decreased apoptosis of VSMCs leading to intimal hyperplasia via uPAR-independent pathways.

The increased expression of uPA in SSc, which we found in the present study, is also supported by other studies. ${ }^{35} 36$ Recently, increased secretion of uPA protein from limited 
A

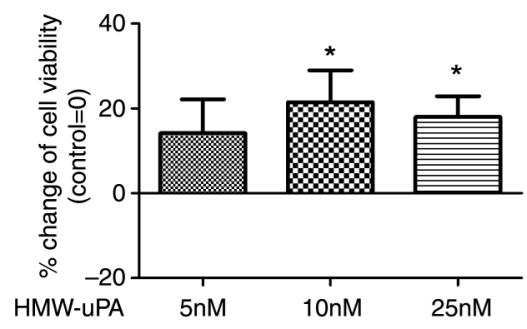

C

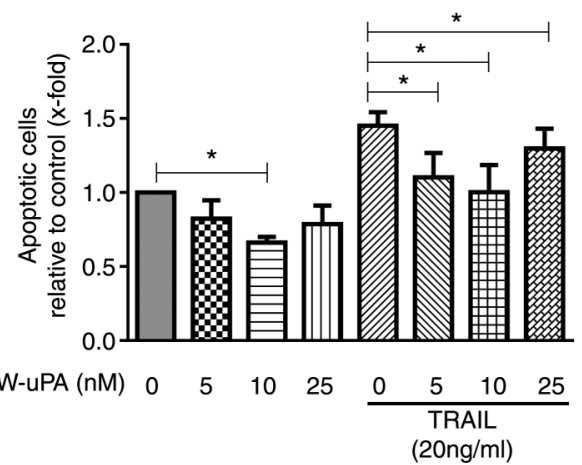

B

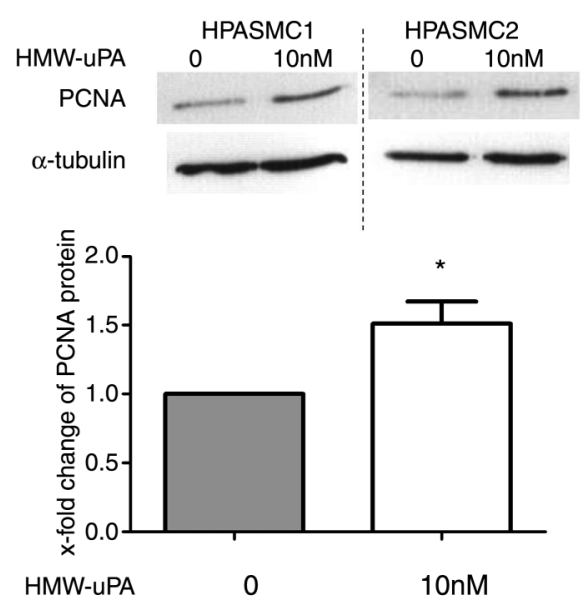

HMW-uPA

$10 \mathrm{nM}$

Figure 5 Urokinase-type plasminogen activator (uPA) increased the proliferation and decreases apoptosis of human pulmonary artery smooth muscle cells (HPASMCs). (A) HPASMCs were stimulated with $5 \mathrm{nM}, 10 \mathrm{nM}, 25 \mathrm{nM}$ high molecular weight uPA (HMW-uPA) for $24 \mathrm{~h}$, and proliferation was assessed by water-soluble tetrazolium salt (WST)-1 assay. Values are reported in percentages of increase of cell viability as compared with unstimulated controls considered as $0 \%$. Values are given as means \pm SEM. Results are from at least five independent experiments. (B) HPASMCs were stimulated with HMW-uPA at $10 \mathrm{nM}$ for $24 \mathrm{~h}$ and proliferating cell nuclear antigen (PCNA) protein was determined by western blotting (top). Representative results are shown. The semiquantitative analysis was performed by densitometry (bottom). Values are given as means ${ }_{ \pm}$SEM. In the western blots, $\alpha$-tubulin was used as loading control. Expression of PCNA in stimulated samples was determined relative to the unstimulated controls, which were defined as 1 . Results are from at least five independent experiments. ${ }^{*} \mathrm{p}<0.05$ versus unstimulated controls. (C) HPASMCs were stimulated with several doses of HMW-uPA with or without TNF-related apoptosis-inducing ligand (TRAIL) (20 ng/mL) for $24 \mathrm{~h}$. Flow cytometry was used to measure levels of propidium iodide (PI)-negative and annexin V-FITC-positive cells relative to controls. Apoptotic cells were defined as PI-negative (indicating an intact plasma membrane) and annexin V-FITC-positive. Values are given as relative changes in the number of PI-negative and annexin V-FITC-positive cells compared with controls. Values are given as means \pm SEM. Results are from at least five independent experiments. ${ }^{*} p<0.05$ versus respective controls.

A

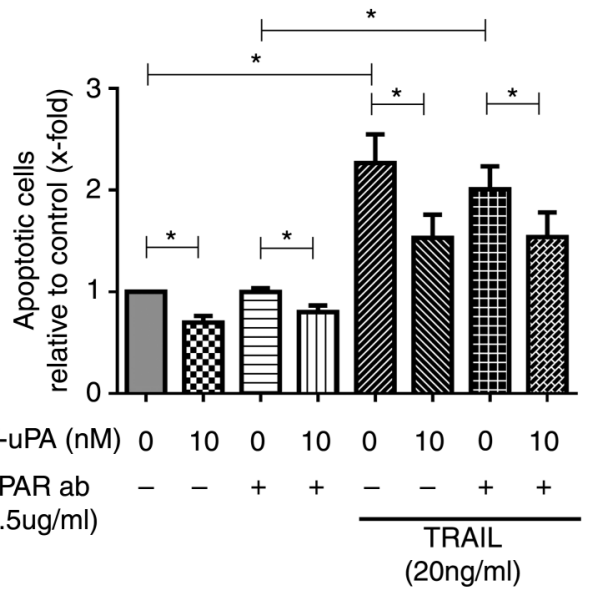

B

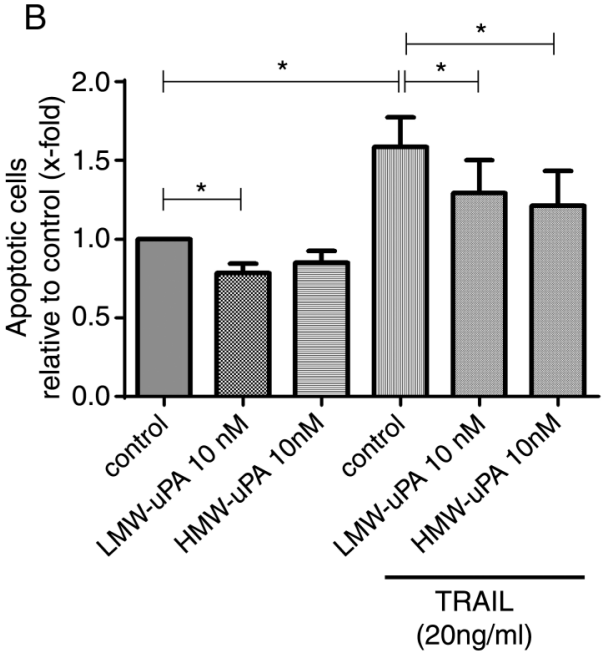

Figure 6 Urokinase-type plasminogen activator (uPA) suppressed apoptosis in human pulmonary artery smooth muscle cells (HPASMCs) via uPA receptor (uPAR) independent pathways. (A) HPASMCs were stimulated with high molecular weight uPA (HMW-uPA) (10 nM), with or without TNF-related apoptosis-inducing ligand (TRAIL) $(20 \mathrm{ng} / \mathrm{mL})$ and neutralising monoclonal antibody against human uPAR $(1.5 \mu \mathrm{g} / \mathrm{mL})$ for $24 \mathrm{~h}$. (B) HPASMCs were stimulated with or without TRAIL $(20 \mathrm{ng} / \mathrm{mL}$ ) and low molecular weight uPA (LMW-uPA) (10 nM) or HMW-uPA (10 nM) for $24 \mathrm{~h}$. Flow cytometry was used to measure levels of propidium iodide (PI)-negative and annexin V-FITC-positive cells relative to control. Apoptotic cells were defined as PI-negative (indicating an intact plasma membrane) and annexin V-FITC-positive. Values are given as relative changes in the number of PI-negative and annexin V-FITC-positive cells compared with unstimulated control. Values are shown as means \pm SEM. Results are from at least five independent experiments. ${ }^{*} p<0.05$ versus respective controls. 
cutaneous SSc fibroblasts compared with normal fibroblasts has been reported. ${ }^{36}$ We could also establish another link between TGF- $\beta$ and vascular changes in SSc, as TGF- $\beta$ strongly enhanced the differential expression of uPA between $\mathrm{SSc}$ and healthy controls.

This is the first study to establish a mechanistic link between deregulation of miRNAs and the proliferative vasculopathy in SSc. We found that miR-193b was significantly downregulated in SSc. Although the role of miR-193b in human disease has not been widely addressed, several studies on miR-193b have been reported in different types of cancers. ${ }^{37-40}$ For example, miR-193b was downregulated in breast cancer, and downregulation of miR-193b increased expression of uPA via direct gene regulation. ${ }^{39}$ Targeting of deregulated miRNA is expected to be a well-tolerated therapy, because differentially expressed miRNAs are often disease-specific and do not play major roles under normal physiological conditions in adult tissues or in quiescent cells. ${ }^{41}$ In fact, miRNA knockout mice often don't display a specific phenotype, unless they are challenged with a stimulus causing cellular stress in target organs. ${ }^{42}$ Our main hypothesis derived from the present study is that increasing levels of miR-193b as a post-transcriptional regulator of uPA could be a therapeutic strategy for the vasculopathy in SSc. This attractive hypothesis needs to be further tested in animal models, and strategies to apply miRNA precursors or mature miRs in vivo have to be developed.

In conclusion, our study showed that downregulation of miR-193b in SSc leads to increased production of uPA, which is a key player in the pathogenesis of SSc. uPA increases the number of pulmonary artery smooth muscle cells and thereby contributes to the proliferative vasculopathy characteristic for SSc. Therefore, strategies to maintain the expression or to prevent the repression of miR-193b have the potential to become a possible therapeutic strategy for the proliferative vasculopathy in SSc.

Acknowledgements The authors thank Maria Comazzi for the excellent technical support.

Contributors NI, OD: conception and design of the study, analysis and interpretation of data and drafting the article. SV: experimental conception and design, miR-expression experiment, hypoxia experiment. MB: experimental conception and design, microRNA microarray experiment. MLW: analysis gene expression microarray data. MC: supply clinical samples. SV, BM, MB, AJ, MC, REG, SG, MLW, JHWD: analysis and interpretation of data, clinical revision of the manuscript. All authors have given their final approval for the manuscript to be published as presented.

Funding This study was supported by an Orphan Disease Program Grant from the European League Against Rheumatism (EULAR).

Competing interests OD has/had consultancy relationship and/or has received research funding in the area of systemic sclerosis and related conditions from Actelion, Pfizer, Ergonex, BMS, Sanofi-Aventis, United BioSource Corporation, Roche/ Genentech, Medac, Biovitrium, Boehringer Ingelheim Pharma, Novartis, 4D Science, Active Biotec, Bayer-Schering, Sinoxa, Serodapharm, EpiPharm, GSK and Biogen. JHWD has consultancy relationships and/or has received research funding from Actelion, Pfizer, Ergonex, BMS, Celgene, Bayer Pharma, Boehringer Ingelheim, JB Therapeutics, Sanofi-Aventis, Novartis, Array Biopharma, KaroBio and Active Biotech in the area of potential treatments of scleroderma and is stock owner of 4D Science $\mathrm{GmbH}$. AJ is funded by the EU-project IMI BTCure. MLW filed patents for gene expression biomarkers in systemic sclerosis and is a scientific founder of Celdara Medical. The real or perceived potential conflicts listed above are accurately stated.

Ethics approval The local ethics committees.

Provenance and peer review Not commissioned; externally peer reviewed.

\section{REFERENCES}

1 Mayes MD, Lacey JV Jr, Beebe-Dimmer J, et al. Prevalence, incidence, survival, and disease characteristics of systemic sclerosis in a large US population. Arthritis Rheum 2003;48:2246-55.
2 Abraham DJ, Krieg T, Distler J, et al. Overview of pathogenesis of systemic sclerosis. Rheumatology (Oxford) 2009;48(Suppl 3):iii3-7.

3 Gabrielli A, Avvedimento EV, Krieg T. Scleroderma. N Engl J Med 2009:360:1989-2003

4 Veale DJ, Collidge TA, Belch JJ. Increased prevalence of symptomatic macrovascular disease in systemic sclerosis. Ann Rheum Dis 1995;54:853-5.

5 Youssef $P$, Englert $H$, Bertouch J. Large vessel occlusive disease associated with CREST syndrome and scleroderma. Ann Rheum Dis 1993;52:464-6.

6 Alfano D, Franco P, Vocca I, et al. The urokinase plasminogen activator and its receptor: role in cell growth and apoptosis. Thromb Haemost 2005:93:205-11.

7 Blasi F, Carmeliet P. uPAR: a versatile signalling orchestrator. Nat Rev Mol Cell Biol 2002:3:932-43.

8 Kjoller L. The urokinase plasminogen activator receptor in the regulation of the actin cytoskeleton and cell motility. Biol Chem 2002;383:5-19.

9 Tkachuk VA, Plekhanova OS, Parfyonova YV. Regulation of arterial remodeling and angiogenesis by urokinase-type plasminogen activator. Can J Physiol Pharmacol 2009;87:231-51.

10 Parfyonova $Y$, Plekhanova O, Solomatina $\mathrm{M}$, et al. Contrasting effects of urokinase and tissue-type plasminogen activators on neointima formation and vessel remodelling after arterial injury. J Vasc Res 2004;41:268-76.

11 Plekhanova OS, Parfyonova YV, Bibilashvily R, et al. Urokinase plasminogen activator enhances neointima growth and reduces lumen size in injured carotid arteries. J Hypertens 2000;18:1065-9.

12 D'Alessio S, Fibbi G, Cinelli M, et al. Matrix metalloproteinase 12-dependent cleavage of urokinase receptor in systemic sclerosis microvascular endothelial cells results in impaired angiogenesis. Arthritis Rheum 2004;50:3275-85.

13 Margheri F, Manetti M, Serrati S, et al. Domain 1 of the urokinase-type plasminogen activator receptor is required for its morphologic and functional, beta2 integrin-mediated connection with actin cytoskeleton in human microvascular endothelial cells: failure of association in systemic sclerosis endothelial cells. Arthritis Rheum 2006:54:3926-38.

14 Manetti M, Rosa I, Milia AF, et al. Inactivation of urokinase-type plasminogen activator receptor (UPAR) gene induces dermal and pulmonary fibrosis and peripheral microvasculopathy in mice: a new model of experimental scleroderma? Ann Rheum Dis 2014;73:1700-9.

15 Axtell MJ. Evolution of microRNAs and their targets: are all microRNAs biologically relevant? Biochim Biophys Acta 2008;1779:725-34.

16 Olena AF, Patton JG. Genomic organization of microRNAs. J Cell Physiol 2010;222:540-5

17 Lewis BP, Shih $\mathrm{IH}$, Jones-Rhoades MW, et al. Prediction of mammalian microRNA targets. Cell 2003;115:787-98.

18 Han M, Toli J, Abdellatif M. MicroRNAs in the cardiovascular system. Curr Opin Cardiol 2011:26:181-9.

19 Lovat F, Valeri N, Croce CM. MicroRNAs in the pathogenesis of cancer. Semin Oncol 2011;38:724-33.

20 Wilmott JS, Zhang XD, Hersey $P$, et al. The emerging important role of microRNAs in the pathogenesis, diagnosis and treatment of human cancers. Pathology 2011;43:657-71.

21 Schetter AJ, Leung SY, Sohn JJ, et al. MicroRNA expression profiles associated with prognosis and therapeutic outcome in colon adenocarcinoma. JAMA 2008:299:425-36.

22 Li H, Yang R, Fan X, et al. MicroRNA array analysis of microRNAs related to systemic scleroderma. Rheumatol Int 2012;32:307-13.

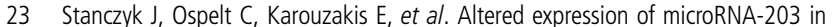
rheumatoid arthritis synovial fibroblasts and its role in fibroblast activation. Arthritis Rheum 2011;63:373-81.

24 Maurer B, Stanczyk J, Jungel A, et al. MicroRNA-29, a key regulator of collagen expression in systemic sclerosis. Arthritis Rheum 2010;62:1733-43.

25 LeRoy EC, Black C, Fleischmajer $R$, et al. Scleroderma (systemic sclerosis): classification, subsets and pathogenesis. J Rheumatol 1988;15:202-5.

26 Distler JH, Wenger RH, Gassmann M, et al. Physiologic responses to hypoxia and implications for hypoxia-inducible factors in the pathogenesis of rheumatoid arthritis. Arthritis Rheum 2004;50:10-23.

27 Niederer F, Ospelt C, Brentano F, et al. SIRT1 overexpression in the rheumatoid arthritis synovium contributes to proinflammatory cytokine production and apoptosis resistance. Ann Rheum Dis 2011;70:1866-73.

28 Distler JH, Jungel A, Huber LC, et al. Imatinib mesylate reduces production of extracellular matrix and prevents development of experimental dermal fibrosis. Arthritis Rheum 2007:56:311-22.

29 Unno K, Zhou Y, Zimmerman T, et al. Identification of a novel microRNA cluster miR-193b-365 in multiple myeloma. Leuk Lymphoma 2009; 50:1865-71.

30 Distler $\mathrm{JH}$, Jungel $\mathrm{A}$, Pileckyte $\mathrm{M}$, et al. Hypoxia-induced increase in the production of extracellular matrix proteins in systemic sclerosis. Arthritis Rheum 2007;56:4203-15.

31 Milano A, Pendergrass SA, Sargent JL, et al. Molecular subsets in the gene expression signatures of scleroderma skin. PLOS ONE 2008;3:e2696. 


\section{Basic and translational research}

32 McMurtry MS, Archer SL, Altieri DC, et al. Gene therapy targeting survivin selectively induces pulmonary vascular apoptosis and reverses pulmonary arterial hypertension. J Clin Invest 2005;115:1479-91.

33 McMurtry MS, Bonnet S, Wu X, et al. Dichloroacetate prevents and reverses pulmonary hypertension by inducing pulmonary artery smooth muscle cell apoptosis. Circ Res 2004:95:830-40.

34 Maglott D, Ostell J, Pruitt KD, et al. Entrez Gene: gene-centered information at NCBI. Nucleic Acids Res 2005;33:D54-8.

35 Bandinelli F, Bartoli F, Perfetto E, et al. The fibrinolytic system components are increased in systemic sclerosis and modulated by Alprostadil (alpha1 ciclodestryn) Clin Exp Rheumatol 2005;23:671-7.

36 Postiglione L, Montuori N, Riccio A, et al. The plasminogen activator system in fibroblasts from systemic sclerosis. Int I Immunopathol Pharmacol 2010;23:891-900.
37 Chen J, Zhang X, Lentz C, et al. miR-193b Regulates Mcl-1 in Melanoma. Am J Pathol 2011;179:2162-8.

38 Leivonen SK, Rokka A, Ostling P, et al. Identification of miR-193b targets in breast cancer cells and systems biological analysis of their functional impact. Mol Cell Proteomics 2011;10:M110 005322.

39 Li XF, Yan PJ, Shao ZM. Downregulation of miR-193b contributes to enhance urokinase-type plasminogen activator (UPA) expression and tumor progression and invasion in human breast cancer. Oncogene 2009;28:3937-48.

40 Rauhala HE, Jalava SE, Isotalo J, et al. miR-193b is an epigenetically regulated putative tumor suppressor in prostate cancer. Int I Cancer 2010;127:1363-72

41 Broderick JA, Zamore PD. MicroRNA therapeutics. Gene Ther 2011;18:1104-10.

42 Thum T, Gross C, Fiedler J, et al. MicroRNA-21 contributes to myocardial disease by stimulating MAP kinase signalling in fibroblasts. Nature 2008;456:980-4. 


\section{$A R D$ Downregulation of miR-193b in systemic sclerosis regulates the proliferative vasculopathy by urokinase-type plasminogen activator expression}

Naoki Iwamoto, Serena Vettori, Britta Maurer, Matthias Brock, Elena Pachera, Astrid Jüngel, Maurizio Calcagni, Renate E Gay, Michael L Whitfield, Jörg H W Distler, Steffen Gay and Oliver Distler

Ann Rheum Dis2016 75: 303-310 originally published online November 10,2014

doi: 10.1136/annrheumdis-2014-205326

Updated information and services can be found at:

http://ard.bmj.com/content/75/1/303

These include:

Supplementary Supplementary material can be found at:

Material http://ard.bmj.com/content/suppl/2014/11/10/annrheumdis-2014-2053 26.DC1

References This article cites 42 articles, 5 of which you can access for free at: http://ard.bmj.com/content/75/1/303\#ref-list-1

Email alerting Receive free email alerts when new articles cite this article. Sign up in the service box at the top right corner of the online article.

Topic
Collections

Articles on similar topics can be found in the following collections

Connective tissue disease (4253)

Immunology (including allergy) (5144)

Pathology (444)

Clinical diagnostic tests (1282)

Genetics (969)

Radiology (1113)

Surgical diagnostic tests (431)

Notes

To request permissions go to:

http://group.bmj.com/group/rights-licensing/permissions

To order reprints go to:

http://journals.bmj.com/cgi/reprintform

To subscribe to BMJ go to:

http://group.bmj.com/subscribe/ 